\title{
Heading Disorientation: A New Test and a Possible Underlying Mechanism
}

\author{
Ritsuo Hashimoto $^{a}$ Yasufumi Tanaka $^{\mathrm{b}}$ Imaharu Nakano ${ }^{c}$ \\ a Department of Neurology, International University of Health and Welfare Hospital, Nasushiobara, bShion Clinic, \\ and ' Division of Neurology, Department of Medicine, Jichi Medical University, Shimotsuke, Japan
}

\section{Key Words}

Topographical disorientation - Heading disorientation • Egocentric reference frame $\cdot$ Directional signal .

Retrosplenial cortex

\begin{abstract}
Background/Aims: Heading disorientation (HD) is a type of pure topographical disorientation. Reported cases showing HD have been very few so far, and its underlying mechanism remains unclear. The aim of this study is to find the anatomic substrates of $\mathrm{HD}$ and to demonstrate a possible underlying mechanism for the symptom. Methods: We investigated 3 patients with HD by a new test, the card-placing test (CPT). Part A of the test assesses the ability of a subject to retain information on spatial locations of cards placed on the floor around the subject. Part B examines the subject's ability to integrate information on the spatial locations of similarly arranged cards and that on changes of the body directions. Results: The patients had a lesion that commonly involved the right retrosplenial cortex and showed good record results for part A but very poor ones for part B. Conclusions: The results of the CPT suggest that HD patients cannot integrate information on the spatial locations of objects derived from an egocentric reference frame with that on changes of the body directions. The retrosplenial cortex may be the place where these different types of information necessary for navigation converge.

Copyright $\odot 2010$ S. Karger AG, Basel
\end{abstract}

\section{Introduction}

Topographical disorientation is defined as the loss of the ability to find one's way within one's locomotor environment. This symptom is often observed in patients with diffuse brain damage (e.g. dementia and acute confusional state). There are certain individuals, however, who show topographical disorientation after focal brain damage. By reviewing such cases comprehensively, Aguirre and D'Esposito [1] recently proposed that there are at least four varieties of topographical disorientation: egocentric disorientation, landmark agnosia, anterograde disorientation, and heading disorientation (HD).

Patients with egocentric disorientation, although they have intact visual recognition ability, exhibit severe deficits as to the relative locations of objects with respect to the self [2-5]. Their performance is often impaired in a wide range of visuospatial tasks such as mental rotation and spatial tapping tasks. Thus, their disability includes not only the representation of a large-scale environment but also wide varieties of cognition that need spatial representational function. Such patients usually have a lesion in the right or bilateral posterior parietal region(s) [2-5].

In contrast to egocentric disorientation, patients with landmark agnosia retain the ability to identify and to represent the locations of objects, but are defective in recognizing salient or prominent environmental features for

\section{KARGER}

Fax +41613061234

E-Mail karger@karger.ch

www.karger.com
(C) 2010 S. Karger AG, Basel

$0014-3022 / 10 / 0632-0087 \$ 26.00 / 0$

Accessible online at:

www.karger.com/ene
Ritsuo Hashimoto, MD

Department of Neurology, International University of Health and Welfare Hospital

537-3 Iguchi, Nasushiobara (Japan)

Tel. +81 28739 3060, Fax +81 287393001

E-Mail ritsuo@iuhw.ac.jp 
a

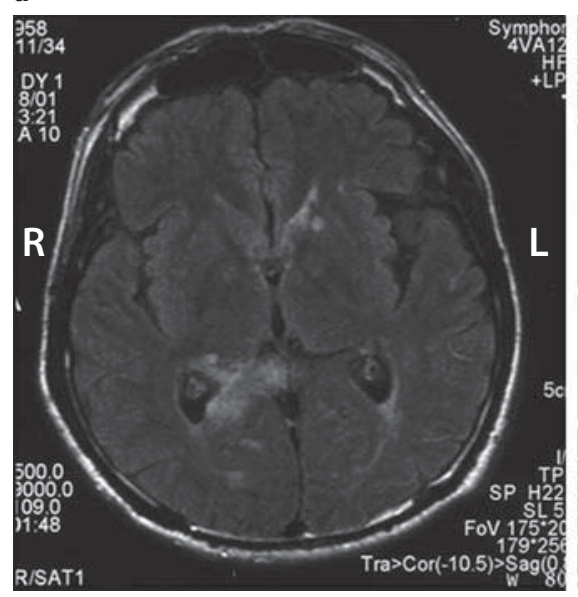

b

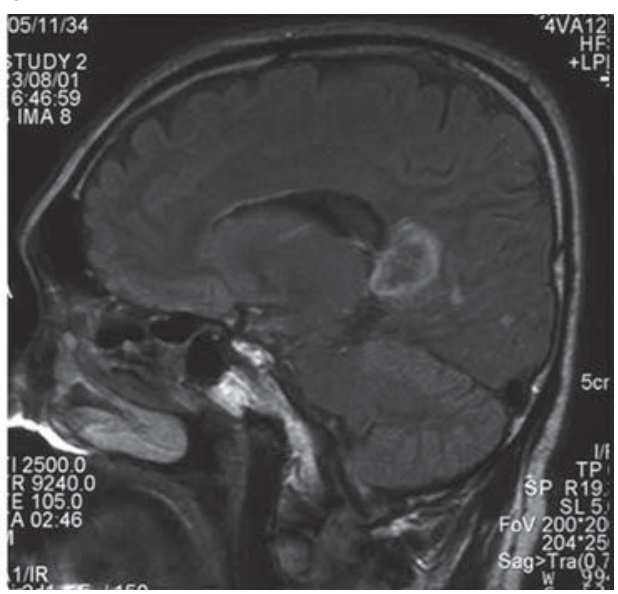

Fig. 1. Fluid attenuated inversion recovery (FLAIR) MRI scan of case 1 (a, axial; b, sagittal views). High signal intensity can be seen in the right retrosplenial area that extends into the right splenium and forceps major of the corpus callosum. the purposes of orientation [6-9]. They usually have a lesion that involves the right or bilateral lingual gyrus, this being very close to the anatomical substrates of patients with visual agnosia whose visual recognition deficits are not confined to landmarks [6-9].

In anterograde disorientation, only impairment in a novel environment is seen, the ability to navigate in familiar places being good. The lesion site responsible for this is presumed to be the parahippocampal gyrus, which is supposed to have some computational function necessary for the acquisition of novel topographical knowledge [10].

HD is different from the above-mentioned three varieties in that HD patients can represent the relative locations of objects and they are able to easily recognize landmarks, but suffer from topographical impairment not only in a novel but also in a familiar environment. It is implied that the primary deficit in HD is an inability to derive directional information from landmarks to reach a destination $[1,11]$.

$\mathrm{HD}$ is thus a very unique and intriguing symptom, however the number of reported cases has been limited so far and a specific test for HD has not been developed yet. Therefore, the underlying mechanism of HD remains unclear. Here, we report $3 \mathrm{HD}$ patients examined by using a new clinical test that we had developed, and discuss a possible underlying mechanism of $\mathrm{HD}$.

\section{Case Reports}

\section{Patient 1}

On August 12, 2001, a 67-year-old right-handed salesman with 9 years of education, who had been under treatment for diabetes mellitus and hypertension for 10 years, was driving a car on his usual way back to home. When he stopped at a crossroad, he suddenly became embarrassed. In the front, he saw buildings and trees that were familiar to him. He was aware that he was near his house, but was unable to determine which direction he should drive. After having made many wrong turns along the way, he managed to finally arrive at his house; it had taken him $2 \mathrm{~h}$ from a place where he usually only needed $5 \mathrm{~min}$ by car. Nine days later, he was referred to our hospital.

On admission, he was alert, cooperative, and neurologically normal including the visual field and visual acuity. He did not show any visual agnosias such as object agnosia or prosopagnosia. The line bisection and line cancellation tasks revealed no hemispatial neglect. His language function was intact. He could repeat 6 digits forward and 5 digits backward. He could tap 6 points forward and 5 backward. His episodic memory function seemed to be intact, because he could remember events that had occurred before, during, and after the onset of his topographical disorientation. During his 1-month stay at our hospital, he frequently lost his way to his room. He said that he had difficulty in remembering the positional relationship of the nurse station, toilets, laboratories and his room. Indeed, even when he could recognize that he was near his room by identifying the nurse station, he could not determine which direction he should go to return back to his room. Although he could say the names of stores or offices near his house, he was unable to draw a map of the vicinity of his house. On the Wechsler Adult Intelligence Scale-Revised (WAIS-R), his verbal IQ was 100, performance IQ was 91, and total IQ was 97. His verbal memory quotient on the Wechsler Memory Scale-Revised (WMS-R, Japanese version) was 110 and that of visual memory was 63.

MRI performed 2 days after his admission revealed an ischemic cerebral lesion at the right retrosplenial area that extended into the right splenium and forceps major of the corpus callosum (fig. 1). His topographical disorientation gradually ameliorated and had almost disappeared 6 months after the onset of his stroke.

\section{Patient 2}

This 72-year-old right-handed man with 9 years of education had a history of cardiac arrhythmia. On May 13, 2004, when he was riding a bicycle, he suddenly lost his way back home. He rec- 
a

Fig. 2. FLAIR MRI scan of case 2 (a, axial; b, sagittal views). High signal intensity can be seen in the right medial occipital lobe that extends into the right retrosplenial area. Low signal intensity that suggests an old infarction can also be seen in the right frontal lobe.

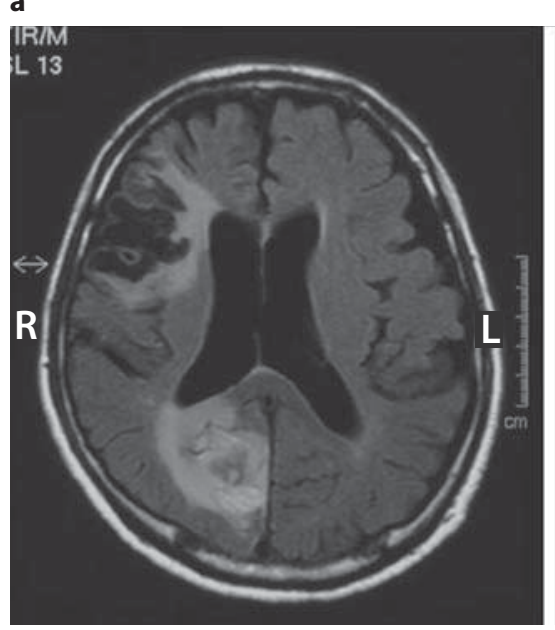

b

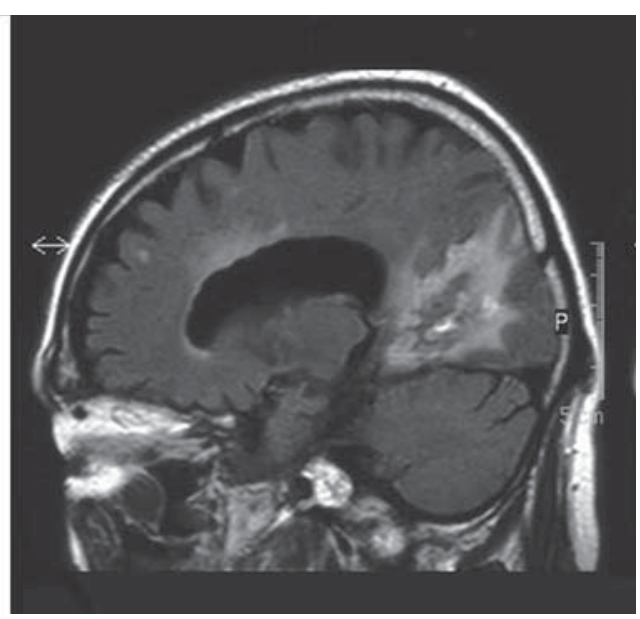

ognized that he was in front of a store near to his house, but was unable to recall the direction his house was in. He needed $30 \mathrm{~min}$ to get back home from a place where he usually needed $5 \mathrm{~min}$ by bicycle. He remembered that he made many mistakes when turning and finally arrived at his house only by spotting familiar landmarks. Two days later, he was referred to our hospital.

On admission, he was alert and cooperative. He had atrial fibrillation but no signs of heart failure. He had left homonymous hemianopsia, but did not show obvious left hemispatial neglect, judging from the line bisection and line cancellation tasks. He did not show any visual agnosias such as object agnosia or prosopagnosia. His language function was intact. He could repeat 5 digits forward and 4 backward. His episodic memory function was thought to be preserved because he could relate things that he had experienced before, during, and after the onset of his topographical disorientation. During his stay at our hospital for 1 month, he had a great trouble in remembering the location of his room and often got lost, even when returning from the toilet that was only $10 \mathrm{~m}$ away from his room. On the WAIS-R, his verbal IQ was 82, performance IQ was 79, and total IQ was 79. In the Rey-Osterrieth Complex Figure Test (ROCFT), his score for copying was $36 / 36$, with $12 / 36$ for immediate recall and $6 / 36$ for delayed recall (30 min after the copying).

MRI on the admission day revealed a fresh infarction in the medial aspect of the right occipital lobe that extended into the right retrosplenial area, and an old infarction in the right frontal lobe (fig. 2). His topographical disorientation gradually improved, but he still had some trouble even 6 months after the stroke.

\section{Patient 3}

This 67-year-old right-handed man with 9 years of education had undergone an operation, partial resectioning of the left lung, because of lung cancer on April 2, 2005. Two days after the operation, he suddenly became unable to return to his room from a toilet that was about $20 \mathrm{~m}$ away. During the following several days, he was often found wandering about the surgical ward looking for his room. Two weeks after the operation, he was referred to the hospital's neurology department.

On the initial neurological examination, he was alert and cooperative. General neurological examination showed that he was intact including the visual acuity and visual field. His language function was normal. He showed no visual agnosias. The line bisection and line cancellation tasks demonstrated that he had no spatial neglect. On the Mini-Mental Status Examination (MMSE, Japanese version), his score was 26/30. In the Auditory Verbal Learning Test (Japanese version), his performance was presumed to be normal because he could say 11 out of 15 words on the fifth recall and remembered 8 of them after a 30 -min delay. His episodic memory function appeared to be preserved because he could say why and when he had received the operation and could relate episodes when he got lost in the surgical ward. In the ROCFT, his scores for copying, immediate recall and delayed recall (30 min after the copying), were $34 / 36,7.5 / 36$ and $4 / 36$, respectively.

MRI on his reference to the neurological department revealed a fresh infarction in the medial aspect of the right occipital lobe that extended into the right retrosplenial area (fig. 3).

\section{Card-Placing Test}

We investigated these 3 patients using a new test, which we named the card-placing test (CPT). The CPT examines the ability to represent spatial locations of objects placed on the floor around a subject. The CPT consists of two parts - part A and B. In part $A$, a subject stands in the center square of nine, $3 \times 3$ each $35 \times 35 \mathrm{~cm}$ drawn on the floor. The subject is instructed to remember locations of three cards with different signs (circle, triangle and cross), each randomly placed in one of the eight squares surrounding the subject. After $10 \mathrm{~s}$, the examiner takes away all the cards, hands them to the subject, and orders the subject to put them back in their original positions. In part B, the subject has to remember the locations of the same three cards placed on the floor as in part A. Immediately after the three cards have been removed, the subject is rotated to the right or to the left by 90 or $180^{\circ}$, as determined in advance (fig. 4), and then asked to restore the three cards to the same locations as before. For each of part A and part $\mathrm{B}$ of the CPT, a subject undergoes 10 consecutive trials. The subject gets 1 point each time he puts one card in the right place and thus the full scores for each of part A and part B are 30 points. 
a

Fig. 3. FLAIR MRI scan of case 3 (a, axial; b, sagittal views). High signal intensity can be seen in the right medial occipital lobe that extends into the retrosplenial area.

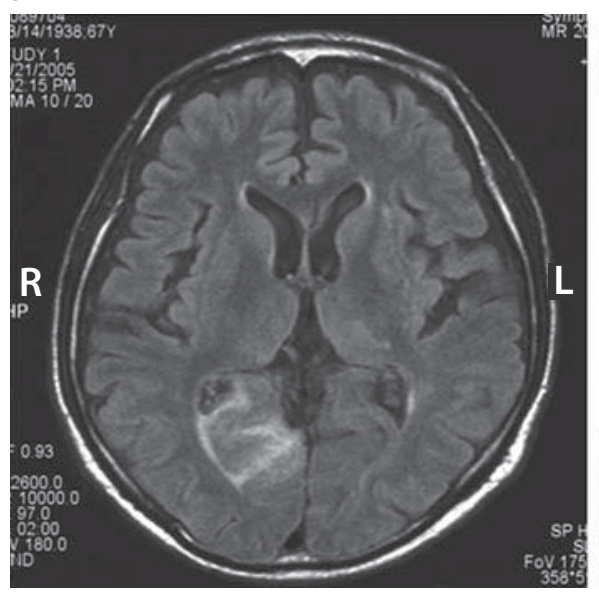

b

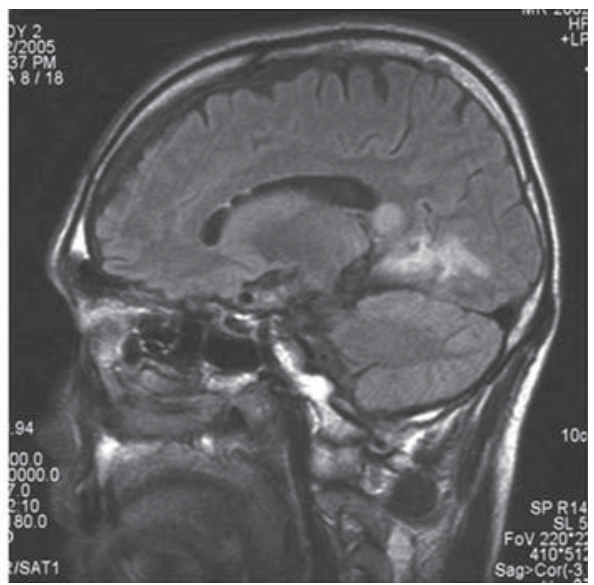

1

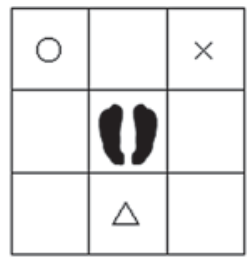

5

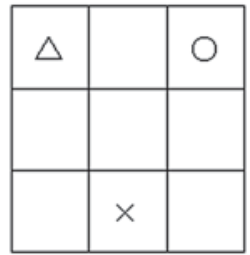

9

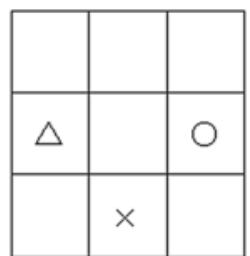

2

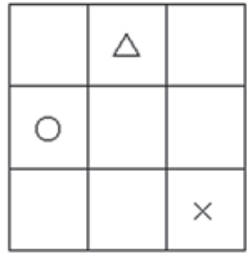

6

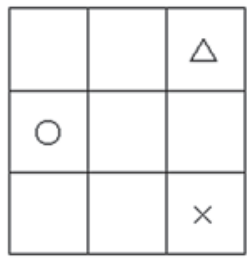

10

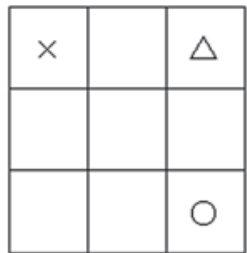

3

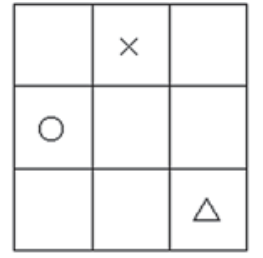

7

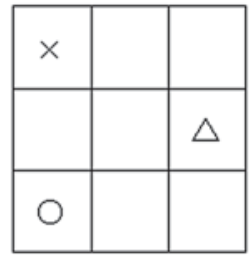

4

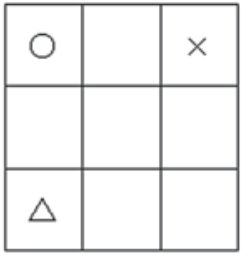

8

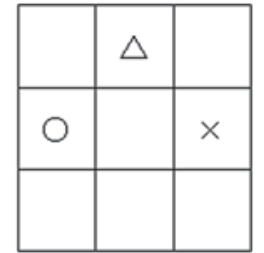

Fig. 4. Card-placing test (CPT). In part $\mathrm{A}$ of the $\mathrm{CPT}$, a subject stands in the center square of nine, $3 \times 3$ squares, drawn on the floor. The subject is instructed to remember the spatial locations of three different cards (circle, triangle and cross), each of which is randomly placed in one of the eight squares surrounding the subject. After $10 \mathrm{~s}$, all the cards are taken away and the subject is requested to restore them to their original positions. The figure shows the positions of the three cards that we used. In part B, the subject also has to remember the locations of the three cards. The positions of the cards are the same as in part A. Immediately after the cards have been removed, the subject is rotated to the right or to the left by 90 or $180^{\circ}$, and then asked to replace the cards. For both part $\mathrm{A}$ and part $\mathrm{B}$ of the $\mathrm{CPT}$, the subject undergoes 10 consecutive trials. The rotations in trials $1-10$ of part $\mathrm{B}$ are as follows: trial $1,90^{\circ}$ to the right; trial $2,90^{\circ}$ to the left; trial $3,180^{\circ}$ to the right; trial $4,180^{\circ}$ to the left; trial $5,90^{\circ}$ to the right; trial $6,90^{\circ}$ to the left; trial $7,180^{\circ}$ to the right; trial $8,180^{\circ}$ to the left; trial $9,90^{\circ}$ to the right, and trial $10,90^{\circ}$ to the left. The numbers indicate the trial numbers. A subject gets 1 point if the location of a card that the subject has replaced is correct, the full score of each of part A and part $\mathrm{B}$ of the CPT being 30 points. 
Table 1. CPT scores of controls and patients

\begin{tabular}{llll}
\hline & $\begin{array}{l}\text { Age } \\
(\text { mean } \pm \mathrm{SD})\end{array}$ & $\begin{array}{l}\text { CPT part A } \\
(\text { mean } \pm \mathrm{SD}) \\
\text { full scores; 30 }\end{array}$ & $\begin{array}{l}\text { CPT part B } \\
(\text { mean } \pm \mathrm{SD}) \\
\text { full scores; 30 }\end{array}$ \\
\hline Controls $(\mathrm{n}=9)$ & $65.4 \pm 4.8$ & $27.6 \pm 3.0$ & $25.0 \pm 4.3$ \\
Case 1 & 67 & 28 & 4 \\
Case 2 & 72 & 26 & 12 \\
Case 3 & 67 & 26 & 17 \\
\hline
\end{tabular}

\section{CPT Scores of Control Subjects}

We carried out the CPT in 9 healthy right-handed volunteers ( 5 women and 4 men) who had never experienced a brain disease such as cerebrovascular disease, head trauma or epilepsy. Their ages ranged from 60 to $72(65.4 \pm 4.8$ [mean $\pm \mathrm{SD}])$. They were independent in daily life and did not complain of topographical disorientation. All of them scored over 27/30 (29.1 \pm 1.1 [mean $\pm \mathrm{SD}]$ ) in the MMSE (Japanese version). As for the CPT, all of them scored over $21 / 30(27.6 \pm 3.0$ [mean \pm SD] $)$ in part $A$ and over $17 / 30(25.0 \pm 4.3[$ mean $\pm \mathrm{SD}])$ in part $\mathrm{B}$.

\section{CPT Scores of the Three Patients}

We examined all the 3 patients within 2 weeks after their stroke, when they showed marked topographical disorientation. The respective scores of cases 1,2 , and 3 in part A were 28/30, $26 / 30$, and $26 / 30$, and those in part B were $4 / 30,12 / 30$, and $17 / 30$ (table 1).

\section{Discussion}

Our 3 patients demonstrated marked topographical disorientation characterized by the following common features: (i) inability to derive directional information from salient landmarks in spite of their ability to recognize them; (ii) topographical disorientation in a novel as well as a familiar environment; (iii) a mild to moderate visual memory disorder; (iv) absence of prominent visuospatial disorientation, as proved by preserved tapping span, no signs of hemispatial neglect and intact constructional abilities, and (v) fairly well-preserved general intellectual functions. These clinical characteristics are quite similar to those in previous case reports [11-17], and according to Aguirre and D'Esposito [1] this type of topographical disorientation is a type of pure topographical disorientation, namely HD.

With respect to the responsible lesion for the development of $\mathrm{HD}$, our 3 cases each had a localized lesion affecting a specific cortical area in common: case 1 had an infarct in the right retrosplenial area that extended into the right splenium and forceps major of the corpus callosum; case 2 had an old infarction in the right dorsolateral frontal lobe and exhibited his HD when he developed a fresh infarction in the right medial occipital lobe involving the right retrosplenial area, and case 3 also had a single ischemic lesion confined to the right retrosplenial area and medial aspect of the right occipital lobe. Damage to the left retrosplenial has been reported to be associated not with HD but with episodic memory deficits [18-21]. Thus, the findings in our cases combined with recent reviews $[1,22]$ strongly indicate that the right retrosplenial region is the critical site for HD. The differences of symptoms between the right and left retrosplenial lesions would be one of the examples of asymmetric organization of higher cerebral functions in humans; in this case, the left retrosplenial area is more associated with aspects of episodic memory, whereas the right one is preferentially concerned with spatial navigation.

What, then, is the mechanism underlying heading disorientation? In normal circumstances, it is considered that the identification of landmarks, information on their spatial locations, heading orientation, and learning and updating information in response to new environment are integrated and used altogether for navigation. Because previous cases with HD and our 3 cases showed mild to moderate anterograde visual memory disturbance, the deficits may contribute their topographical disorientation especially in a setting of new environment. A case reported by Suzuki et al. [15], however, did not demonstrate visual memory disturbance at all and the patients with HD including ours showed disorientation not only in a new but also in a well-known familiar environment $[1,11-17,22]$. Thus, anterograde visual memory disturbance alone cannot explain their disorientation. Given that patients with HD retain the abilities to recognize landmarks and to map the spatial locations of immediate objects, they should be specifically defective in integrating head directional signals. This is further supported by the results of the CPT in our 3 patients.

To accomplish part A of the test, the subjects are likely to have to use an egocentric reference frame. The normal scores for part $\mathrm{A}$ in our 3 patients suggest that they were able to use an egocentric reference frame to represent the spatial locations of objects surrounding them. In contrast, all 3 patients had great difficulty in performing part B. Although the score of 1 patient (case 3) was borderline, those of the other 2 (cases 1 and 2) were well below the controls'. Moreover, all of them appeared to be truly perplexed when they were engaged in performing part B: an observation that was not seen in control sub- 
jects. The only difference between part A and part B is the subjects' rotation in part $B$ just before they replace the cards. The most plausible explanation for their poor achievement in part $B$ is that the patients were unable to use the information on changes in their body direction. In other words, they were defective in the processing of directional signals of the self, or unable to integrate information on the registered external spatial locations of objects with that on their body direction.

There are lines of evidence that the retrosplenial cortex is concerned with spatial navigation. For example, excision of the retrosplenial cortex of rats can impair spatial navigation $[23,24]$. Recent neuroimaging studies in humans using PET or fMRI also revealed that the retrosplenial area was activated when subjects were involved in a large-scale navigation task, although the activation was usually bilateral [25-27]. Moreover, it is noteworthy that in rats, head direction cells - cells that are excited when rats are maintaining a certain heading or orientation within an environment - are found in the retrosplenial cortex in addition to several neural structures such as the anterior dorsal nucleus of the thalamus, lateral dorsal thalamus, lateral mammillary nuclei, striatum, and postsubiculum $[28,29]$. These neural substrates may constitute a functional circuit dealing with directional signals of the self [28].
Several experiments in monkeys have shown that the retrosplenial cortex is reciprocally connected with the parahippocampal cortical areas, presubiculum, entorhinal cortex, mid-dorsolateral prefrontal cortex, superior temporal sulcus, and posterior parietal cortex [30-32]. Therefore, the retrosplenial cortex seems to be a pivotal constituent of both the medial temporal lobe memory system and head directional system as well as interacting with the lateral parietofrontal large-scale network, which contributes to the processing of information on the egocentric visuospatial locations of objects.

In conclusion, our 3 cases suggest that the right retrosplenial lesions were responsible for their HD. The CPT implied that HD patients cannot integrate information well on object locations in the space derived from an egocentric reference frame and that on changes in body direction. The right retrosplenial cortex may be a place where these different types of information necessary for navigation converge. The CPT is a very useful clinical tool for evaluating patients with HD.

\section{Acknowledgements}

We thank Drs. Hiroyuki Kato, Tomoko Ogawa, and Ryohei Hishida for their help in examining the patients. We also thank Ms. Noriyo Komori and Ms. Tomomi Miyazaki for their neuropsychological assessment of the patients.

\section{References}

1 Aguirre GK, D’Esposito M: Topographical disorientation: a synthesis and taxonomy. Brain 1999;122:1613-1628.

2 Kase CS, Troncoso JF, Court JE, Tapia JF, Mohr JP: Global spatial disorientation. Clinico-pathologic correlations. J Neurol Sci 1977;34:267-278

3 Levine DN, Warach J, Farah MJ: Two visual systems in mental imagery: dissociation of 'what' and 'where' in imagery disorders due to bilateral posterior cerebral lesions. Neurology 1985;35:1010-1018.

4 Stark M, Coslett HB, Saffran EM: Impairment of an egocentric map of locations: implications for perception and action. Cogn Neuropsychol 1996;13:481-523.

5 Wilson BA, Berry E, Gracey F, Harrison C, Stow I, Macniven J, Weatherley J, Young AW: Egocentric disorientation following bilateral parietal lobe damage. Cortex 2005;41:547554

6 Hécaen $\mathrm{H}$, Tzortzis $\mathrm{C}$, Rondot P: Loss of topographic memory with learning deficits. Cortex 1980;16:525-542.
7 McCarthy RA, Evans JJ, Hodges JR: Topographical amnesia: spatial memory disorder perceptual dysfunction, or category specific semantic memory impairment? J Neurol Neurosurg Psychiatry 1996;60:318-325.

8 Landis T, Cummings JL, Benson DF, Palmer EP: Loss of topographic familiarity. An environmental agnosia. Arch Neurol 1986;43: 132-136

9 Takahashi N, Kawamura M, Hirayama K, Tagawa K: Non-verbal facial and topographic visual object agnosia - a problem of familiarity in prosopagnosia and topographic disorientation (in Japanese). No To Shinkei 1989;41:703-710.

10 Habib M, Sirigu A: Pure topographical disorientation: a definition and anatomical basis. Cortex 1987;23:73-85.

11 Takahashi N, Kawamura M, Shiota J, Kasahata N, Hirayama K: Pure topographical disorientation due to right retrosplenial lesion. Neurology 1997;49:464-469.

12 Bottini G, Cappa S, Geminiani G, Sterzi R: Topographic disorientation - a case report. Neuropsychologia 1990;28:309-312.
13 Obi T, Bando M, Takeda K, Sakuta M: A case of topographical disturbance following left medial parieto-occipital lobe infarction (in Japanese). Rinsho Shinkeigaku 1992;32: 426-429.

14 Cammalleri R, Gangitano M, D'Amelio M, Raieli V, Raimondo D, Camarda R: Transient topographical amnesia and cingulated cortex damage: a case report. Neuropsychologia 1996;42:321-326.

15 Suzuki K, Yamadori A, Hayakawa Y, Fujii T: Pure topographical disorientation related to dysfunction of the viewpoint dependent visual system. Cortex 1998;34:589-599.

16 Katayama K, Takahashi N, Ogawara K, Hattori T: Pure topographical disorientation due to right posterior cingulated lesion. Cortex 1999;35:279-282.

17 Alsaadi T, Binder JR, Lazar RM, Doorani T, Mohr JP: Pure topographical disorientation: a distinctive syndrome with varied lesion location. Neurology 2000;54:1864-1866. 
18 Valenstein E, Bowers D, Verfaellie M, Heilman KM, Day A, Watson RT: Retrosplenial amnesia. Brain 1987;110:1631-1646.

19 Takayama Y, Kamo H, Ohkawa Y, Akiguchi I, Kimura J: A case of retrosplenial amnesia (in Japanese). Rinsho Shinkeigaku 1991;31: 331-333.

20 Katai S, Maruyama T, Hashimoto T, Yanagisawa N: A case of cerebral infarction presenting as retrosplenial amnesia (in Japanese). Rinsho Shinkeigaku 1992;32:1281-1287.

21 Gainotti G, Aimonti S, Di Betta AM, Silveri MC: Retrograde amnesia in a patient with retrosplenial tumour. Neurocase 1998;4: 519-526.

22 Maguire EA: The retrosplenial contribution to human navigation: a review of lesion and neuroimaging findings. Scand J Psychol 2001;42:225-238.
23 Sutherland RJ, Whishaw IQ, Kolb B: Contributions of the cingulate cortex to two forms of spatial learning and memory. J Neurosci 1988;8:1863-1872.

24 Cooper BG, Mizumori SJY: Retrosplenial cortex inactivation selectively impairs navigation in darkness. Neuroreport 1999;10: 625-630.

25 Aguirre GK, D’Esposito M: Environmental knowledge is subserved by separable dorsal/ ventral neural areas. J Neurosci 1997; 17 2512-2517.

26 Ghaem O, Mellet E, Crivello F, Tzourio N, Mazoyer B, Bethoz A, Denis M: Mental navigation along memorized routes activates the hippocampus, precuneus, and insula. Neuroreport 1997;8:739-744.

27 Maguire EA, Burgress N, Donnet JG, Frakowiak RSJ, Frith CD, O’Keefe J: Knowing where, and getting there: a human navigation network. Science 1998;280:921-924.

28 Taube JS: Head direction cells and the neurophysiological basis of a sense of direction. Prog Neurobiol 1998;55:225-256.
29 Chen LL, Lin LH, Green EJ, Barnes CA, MacNaughton BL: Head-direction cells in the rat posterior cortex. I. Anatomical distribution and behavioral modulation. Exp Brain Res 1994;101:8-23.

30 Kobayashi Y, Amaral D: Macaque monkey retrosplenial cortex. I. Three-dimensional and cytoarchitectonic organization. J Comp Neurol 2000;426:339-365.

31 Suzuki WA, Amaral D: Perirhinal and parahippocampal cortices of the macaque monkey: cortical afferents. J Comp Neurol 1994; 350:497-533.

32 Morris R, Pandya D, Petrides M: Fiber system linking the mid-dorsolateral frontal cortex with the retrosplenial/presubicular region in the rhesus monkey. J Comp Neurol 1999;407:183-192. 
Copyright: S. Karger AG, Basel 2010. Reproduced with the permission of S. Karger AG, Basel. Further reproduction or distribution (electronic or otherwise) is prohibited without permission from the copyright holder. 